\title{
ESTRATEGIAS PARA SACAR VENTAJA DE LAS TECNOLOGIAS DISRUPTIVAS EN EL SECTOR DE LAS TECNOLOGIAS DE LA INFORMACION Y LAS COMUNICACIONES
}

\section{STRATEGIES TO OBTAIN ADVANTAGE OF DISRUPTIVE TECHNOLOGIES IN THE INFORMATION AND COMMUNICATIONS TECHNOLOGY SECTOR}

Esp. Hulber Rodrigo Rodríguez Pinzón.*, MSc. Andrés Mauricio Puentes Velásquez ** Esp. María Liliana Suarez Domínguez **

*Servicio Nacional de Aprendizaje Sena, Tecnoparque Nodo Ocaña, Grupo de Investigación GINDET.

Transversal 30 N 7-110 La Primavera, Ocaña Norte de Santander, Colombia. Teléfono, (57) (7) 5611035

E-Mail: hrrodriguepi@misena.edu.co

*Universidad Francisco de Paula Santander, Facultad de Ingeniería, Programa de Maestría en gobierno de Tecnologías de la Información, Grupo de Investigación GITYD.

Vía Acolsure Sede el Algodonal, Ocaña, Norte de Santander, Colombia. Teléfono, (57) (7) 5690088

E- Mail: ampuentesv@misena.edu.co

Resumen: Este artículo pretende definir una serie de estrategias que le permite a las empresas de telecomunicaciones obtener ventajas de las tecnologías disruptivas que en ocasiones amenazan la permanencia de las empresas del sector. Se plantea hacer un estudio de estándares de TI que puedan ayudar a fortalecer estrategias de negocio, de igual manera una profundización de las áreas de TI que pueden fortalecer ideas innovadoras y de algunas políticas nacionales en materia de tecnología de las cuales se pueda sacar provecho para que las tecnologías disruptivas cambien las empresas de manera positiva. 


\section{INTRODUCCIÓN}

La toma de decisiones es el factor crítico para las organizaciones el cual se hace aún más complejo cuando no se cuenta con las herramientas que permitan facilitar y entender de una manera más organizada los procesos. Los retos empresariales en la actualidad son mucho más grandes y ameritan perfeccionamiento continuo e innovación; para el teórico austríaco Joseph Schumpeter, la innovación no es más que la capacidad para identificar un vacío y desarrollar de manera efectiva una estrategia que permita llenar este vacío como si nunca hubiera existido. (Levy, 2018)

En la actualidad existen herramientas de ayuda para que la administración y gestión empresarial se realicen de mejor manera y que permiten que los negocios avancen al ritmo acelerado de la tecnología y sacar ventaja de ella, El Irlandés Tim O'Reilly, considerado uno de los padres de la web 2.0, creador de la primera tienda de libros digitales en Internet y cofundador de AOL, afirma que en el ecosistema digital solo sobrevive aquel que tiene la capacidad de adaptarse a lo impredecible y cuyo aporte es impactante y disruptivo; de modo que el sector de las telecomunicaciones debe trabajar continuamente para superar obstáculos y mantener sus modelos de negocio. En este orden de ideas a través de este artículo se brindan unas estrategias que pueden ayudar a dichas empresas a obtener ventajas competitivas.

\section{MARCO CONCEPTUAL}

\subsection{Tecnologías disruptivas}

La tecnología disruptiva se puede definir como una innovación que ayuda a crear una nueva red de valor y que eventualmente interrumpe el mercado actual (en unos pocos años o décadas), desplazando una tecnología anterior. (Loza \& Dabirian, 2015)

\subsection{Gestión de TI}

Es una disciplina basada en procesos, enfocada en alinear los servicios de TI proporcionados con las necesidades de las empresas, poniendo énfasis en los beneficios que puede percibir el cliente final. GSTI propone cambiar el paradigma de gestión de TI, por una colección de componentes enfocados en servicios de punta a cabo usando distintos marcos de trabajo con las "mejores prácticas", como por ejemplo la Information Technology Infrastructure Library (Itsqc.org, 2018)

\subsection{Sector TIC.}

El sector de Tecnologías de la Información y las Comunicaciones está conformado por los proveedores de redes y servicios de telecomunicaciones, los proveedores del servicio de radiodifusión sonora y los operadores de servicios postales, (Decreto 1078, 2015).

\subsection{TIC.}

Las Tecnologías de la Información y las Comunicaciones (en adelante TIC), son el conjunto de recursos, herramientas, equipos, programas informáticos, aplicaciones, redes y medios, que permiten la compilación, procesamiento, almacenamiento, transmisión de información como voz, datos, texto, video e imágenes. (Ley 1341, 2009).

\section{ESTRATEGIAS PARA OBTENER VENTAJAS DE LAS TECNOLOGIAS DISRUPTIVAS.}

\subsection{Elegir estratégicamente un CIO Chief Information Officer.}

Elegir de manera idónea la persona que tenga el perfil para transformar de manera positiva una organización y que enfrente las crisis como fuentes para generar modelos de negocio diferentes y que sea consiente que toda labor que emprenda el sector de las telecomunicaciones debe estar dirigido por una persona preparada que se especialice en el tema y que cuente con el conocimiento y la tenacidad que se requiere para la toma de decisiones lo que hoy se conoce como CIO Chief Information Officer (Director de Información) " líderes de la gestión estratégica de Tecnologías de Información, encargados de planificar, organizar, coordinar, gestionar y controlar la estrategia de uso y apropiación de TI, y todo lo que conlleva esta tarea" (Ministerio de Tecnologías de la Información y las Comunicaciones, Mintic, 2018) que conozca buenas prácticas de gerencia, estándares de TI y generación de estrategias de negocio esto ayudara a que las afectaciones para las empresas de 
telecomunicaciones sean menos traumáticas y permitirá brindar servicios que satisfagan al cliente sin afectar el modelo de negocio.

Para hacer parte del sector de las telecomunicaciones se debe estar en contexto con las realidades a nivel mundial y siempre pensando en ir más allá de lo actual porque este sector da pasos agigantados lo que exige que el gerente de estas organizaciones camine a este mismo ritmo; en caso de no ser así los cambios serán traumáticos a nivel operativo, financiero y pondrán en riesgo a la organización.

Por la importancia de la toma de decisiones como estrategia que permita obtener ventajas de la tecnología disruptiva, se dejan las principales características que deberá detectar a la hora de seleccionar el CIO de su organización.

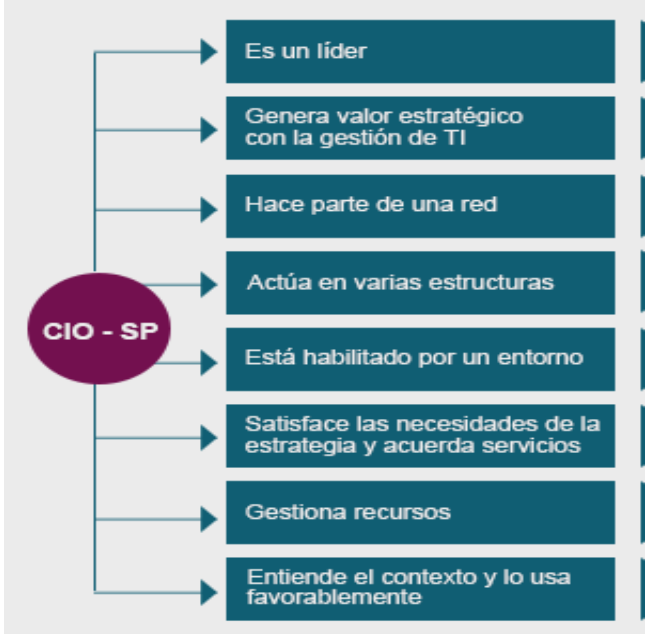

Figura 1. Características de un CIO. Fuente:

Ministerio de información y Comunicaciones

\subsection{Perder el miedo a las tecnologías disruptivas y verlas como innovación.}

Evidentemente, la innovación disruptiva trae consigo mucho más riesgos, razón por la cual suelen ser emprendedores de nuevas aventuras empresariales las que exploran todo su potencial. Las empresas establecidas normalmente no invierten agresivamente en este tipo de innovaciones principalmente porque les falta capacidad para identificar el potencial de la nueva propuesta de valor, debido a que es analizada desde la lógica dominante del negocio, de igual manera, al estar dirigida inicialmente al segmento bajo del mercado, presenta márgenes muy bajos de

\section{Universidad de Pamplona}

I. I. D. T. A. beneficios iniciales. Finalmente, estas innovaciones muchas veces requieren cambios fundamentales en la estructura de la empresa, proveedores, distribución y canales de ventas; lo que cuestiona propuestas de valor actual de la empresa. Sin embargo, el éxito de las innovaciones disruptivas está relacionado con principalmente con:

Perder el miedo: El miedo es el principal factor que limita la innovación; solo cuando se pierde esta barrera se pueden crear nuevas ideas y nuevas estrategias; se debe perder el miedo a intentar nuevos modelos de negocio, nuevas estrategias que feliciten el objeto de la empresa y sobre todo que motiven seguir en crecimiento derribando la disrupción.

Ver a las tecnologías disruptivas como un incentivo de innovación: en esta característica se trae en mención aquel dicho popular "La competencia genera excelencia" precisamente la disrupción genera cambios drásticos para las empresas y puede llegar a generar diversas dificultades cuando no se está preparado; sin embargo es un factor clave para innovar porque ayuda a despertar de la comodidad al empresario y lo obliga a pensar y proyectar su organización de una mejor manera, generación de nuevos servicios, generación de nuevos modelos de negocio e incluso genera cambios culturales por ejemplo el teléfono móvil que genero un cambio en el modo de pensar y la disponibilidad de tiempo de las personas, la facilidad de ubicación y demás factores que desplazo al teléfono fijo.

\subsection{Cómo hacer frente a las innovaciones disruptivas}

Conocimiento de Estándares de TI. El conocimiento de los estándares de TI genera calidad en las estrategias de negocio que puedan aplicar las empresas, la ausencia de dichos estándares impide que las tareas sean realzadas ordenadamente siguiendo faces de planeación organización y control, además de muchos otros factores que estos estándares otorgan. Algunos de los estándares que se recomienda hacer uso en las empresas de telecomunicaciones y que serán una estrategia muy útil para apoyar las decisiones:

COBIT- La misión de Cobit es investigar, desarrollar, hacer público y promover un marco de 
control de gobierno de TI autorizado, actualizado y aceptado internacionalmente, para la adopción, por parte de las empresas y el uso diario, por parte de gerentes de negocio, profesionales de TI (ITGI, 2007)

En el presente artículo se recomienda este estándar partiendo de que una de sus características es que las empresas deben medir dónde se encuentran y dónde se requieren mejoras, e implementar herramientas gerenciales para monitorear dicha mejora; lo que va de la mano de la tarea de hacer frente a la disrupción tecnológica para generar innovaciones que proyecten la organización hacia un modelo de negocio diferente con mayores ventajas.

ISO 38500- proporcionar un marco de principios para que la dirección de las organizaciones lo utilice al evaluar, dirigir y monitorear el uso de TI y obtener resultados que apoyen la toma de decisiones de manera mucho más acertada. ( ISO 38500, 2009)

ITIL- es el conjunto de conceptos y mejores prácticas para la administración de servicios de TI. Todos los estándares mencionados en este documento van dirigidos a proporcionar herramientas para una buena toma de decisiones que es lo que se requiere cuando se necesita transformar modelos de negocio. (Servicetonic, 2018)

Conocimiento de políticas nacionales en materia tecnológica. Es muy común pensar que las políticas no tienen nada que ver con el desarrollo de nuestras organizaciones y que es tarea de los dirigentes del país encargarse de esos asuntos; sin embargo por pensar de esta manera las empresas de telecomunicaciones en Colombia se están volviendo insostenibles porque tienen accionistas que ni siquiera saben que existen y uno de ellos es el estado, la pregunta es ¿Cómo se volvió el estado accionista de las empresas en Colombia? Lo hizo a través de las políticas que implementa a diario para regular las empresas y en este caso el sector de las telecomunicaciones, por ende una de las principales tareas para hacer frente a las tecnologías disruptivas es conocer las políticas en materia de tecnología, sacar sus ventajas y desventajas y participar en los debates para generar mejoras que beneficien el modelo de negocio.
Entre las principales políticas que debe entender y conocer el sector tic esta la ley 1341 de 2009 que regula el sector TIC en Colombia, la ley 1607 que establece normas en materia tributaria $y$ actualmente se debe estar en permanente participación del proyecto de ley que busca la restructuración del sector TIC y que aún no ha sido aprobado en su totalidad.

\subsection{Principales tecnologías disruptivas para el sector tic}

En el área de las telecomunicaciones es donde mayor disrupción existe, cada día surgen nuevas tecnologías que transforman y cambian el mundo; en este artículo se presentaran algunas de ellas que están impactando en la actualidad.

IOT: El concepto de Internet de las cosas que posibilita que Internet alcance el mundo real de los objetos físicos, se refiere a una amplia variedad de tecnologías, sistemas de posicionamiento global, RFID, comunicaciones inalámbricas de corto alcance, localización en tiempo real, y, por supuesto, Internet, combinados para formar una enorme red, con el objetivo de que todas las cosas conectadas con esta red puedan servir dentro de un sistema automatizado para, por ejemplo, la identificación de objetos en tiempo real, la localización, seguimiento, monitoreo y activación de eventos de diversa índole. (Fermín \& Guerra, 2015)

Es clave que las empresas de telecomunicaciones empiecen a empoderarse e ir implementando esta tendencia en sus estrategias de negocio, puesto que "Será una tendencia que transformará todos los mercados e impactará en todas las industrias" declara José Manuel Petisco, director general de Cisco en España. "Moverá 19 billones de dólares en todo el mundo, de los cuales 14,4 billones se generarán en el ámbito privado y 4,6 billones del público". Por lo anteriormente expuesto IOT es otra herramienta para innovar en las telecomunicaciones, a pesar de ser disruptivo

OTT. (Over The Top, por sus siglas en inglés) que hacen referencia a la distribución de contenidos hasta el usuario final haciendo uso de Internet por una fuente diferente a la que provee los servicios 
de acceso a la red. Basado en el principio de neutralidad de red, el cual establece que el proveedor de la conexión a Internet debe dar tratamiento igual a todos los contenidos que viajen por la red, sin dar trato preferencial a los servicios de valor agregado provistos por la misma compañía; esto ha permitido a los servicios OTT competir de manera efectiva. (Cuevas, 2012).

Los contenidos OTT son un factor altamente disruptivo para las empresas de televisión y al hacer parte del sector de tecnología se debe estar preparados a los cambios que a diario ofrecen. No es la primera vez que la industria de la televisión sufre una migración tecnológica de manera disruptiva, ya que existió también el paso de formato de señal analógica blanco y negro a los formatos a color actuales, como el National Television Society Committee (NTSC) americano, el alemán Phase Alternating Line (PAL) (Badillo, 2012)

\section{Trabajos Relacionados}

En esta sección se presentan un conjunto de trabajos relacionados con la presente investigación, los cuales se enmarcan en la temática de las tecnologías disruptivas.

4.1 Estudio realizado por KPMG International con el apoyo de Forrester Consulting titulado "Una llamada a la acción Barómetro de tecnologías disruptivas en el sector de los medios de comunicación"

El estudio realizado comprende preguntas cuantitativas respecto a las principales tendencias de adopción de tecnologías disruptivas dentro del sector TIC, de igual manera la afectación de dichas tecnologías a los modelos de negocio, operaciones, marketing y actividades de cara al cliente de las empresas, también muestra en qué tecnologías disruptivas están invirtiendo las empresas y lo que esperan lograr de dichas inversiones.

4.2 Investigación de EYQ. Las ventajas de la disrupción mega tendencias para el futuro.

Esta investigación muestra como la disrupción está en todos los aspectos de la vida. Como resultado de la rápida aceptación de la innovación disruptiva, la comunidad empresarial está cada vez más consciente de que esta fuerza trasformadora está involucrada en todos los aspectos de la vida y gana terreno a un ritmo sin precedentes, sin embargo, a pesar de que existe una mayor conciencia al respecto, son pocas las compañías que han logrado transformar sus modelos de negocios de manera exitosa. Por ejemplo, Netflix cambió su modelo de negocios de uno basado en la entrega a domicilio de los DVD a uno basado en streaming de contenidos vía internet

\section{CONCLUSIONES}

El conocimiento de los estándares de TI genera calidad en las estrategias de negocio que puedan aplicar las empresas y el desconocimiento de éstos, impide que las tareas sean realzadas ordenadamente siguiendo faces de planeación organización y control, además de muchos otros factores que dichos estándares otorgan

Las compañías deben estar actualizándose permanentemente en materia de normatividad y demás estrategias que puedan influir en el correcto desarrollo del negocio, pensando en una adecuada toma de decisiones.

Las empresas del sector TIC deben generar alternativas de negocio que lleven implícito el toque de personalización y que ataque a segmentos donde las grandes compañías no están interesadas en llegar, de esta manera podrán potencializar sus servicios y debilitar las afectaciones que los pasos agigantados de la tecnología traen, en pocas palabras deben ver las tecnologías disruptivas como una manera de innovar lo que tienen para poder fortalecerse y mantenerse en el mercado.

\section{REFERENCIAS}

ISO 38500. (2009). NTC-ISO/IEC 38500. G. obierno Corporativo de la Tecnología Informática.

Badillo. (2012). Economía política de los estándares de televisión digital: el caso de América Latina. Obtenido de http://www.elsevier.es/es-revista-sumanegocios-208-articulo-climaorganizacional-migracion-tecnologicaapagon-S2215910X15000294\#bib0035 
Cuevas, R. J. (2012). Impacto de la penetración de la banda ancha en la TV abierta y servicios OTT. Instituto Federal de Telecomunicaciones. Centro de Estudios. Obtenido de Impacto de la penetración de la banda ancha en la TV abierta y servicios OTT

Decreto 1078. (2015). Por medio del cual se expide el Decreto Único Reglamentario del Sector de Tecnologías de la Información y las Comunicaciones. Bogota, Colombia, 26 de mayo de 2015. Obtenido de https://www.mintic.gov.co/portal/604/arti cles-9528_documento.pdf

Fermín, P. F., \& Guerra, G. J. (2015). Internet de las Cosas. Obtenido de http://revistas.uigv.edu.pe/index.php/persp ectiva/article/view/187

ITGI. (2007). Cobit 4.1. . Rolling Meadows,.

Itsqc.org. (2018). The Service Quality Model for eSourcing. Obtenido de http://www.itsqc.org/models/escmsp/index.html

Levy, B. G. (2018). Monetización de servicios: el gran desafío digital. Obtenido de http://andinalink.com/monetizacion-deservicios-el-gran-desafio-digital/

Ley 1341. (2009). Recuperado el 6 de mayo de 2018 , de http://www.alcaldiabogota.gov.co/sisjur/n ormas/Norma1.jsp?i=36913

Loza, M. D., \& Dabirian, R. (Septiembre de 2015). Introducción a la Tecnología Disruptiva y su Implementación en Equipos Científicos. Revista Politécnica Vol. 36, No. 3.

Ministerio de Tecnologías de la Información y las Comunicaciones, Mintic. (2018). CIO (Chief Information Officer). Obtenido de https://www.mintic.gov.co/gestionti/615/ w3-propertyvalue-6205.html

Servicetonic. (2018). ¿Qué es ITIL? Obtenido de https://www.servicetonic.es/itil/introducci on-a-itil-v3/ 